Supporting Information

\title{
Dynamics of Crowding-Induced Mixing in Phase Separated Lipid Bilayers
}

\author{
Wade F. Zeno ${ }^{\star}$, Kaitlin E. Johnson*, Darryl Y. Sasaki`, Subhash H. Risbud ${ }^{\dagger}$,
} and Marjorie L. Longo**

${ }^{\star}$ Department of Chemical Engineering, University of California Davis, Davis, California 95616,

\section{United States}

ISandia National Laboratories, P.O. Box 969, Livermore, California 94551, United States

†Department of Materials Science and Engineering, University of California Davis, Davis,

California 95616, United States

$\mathbf{L}_{\mathbf{0}}$ Domain Concentration Profile Derivation. In equation $\mathrm{S} 1$, the one-dimensional, time dependent radial diffusion was approximated in Cartesian coordinates, rather than cylindrical coordinates, since an analytical solution in cylindrical coordinates is difficult to obtain with the given initial and boundary conditions (equations S1a-c).

$$
\begin{array}{rrr}
\frac{\partial C(t, r)}{\partial t} \approx D \frac{\partial^{2} C(t, r)}{\partial r^{2}}, t & \in[0, \infty], r \in\left[R(t), R_{b}\right] \\
C(0, r) & =C_{1} & (\mathrm{~S} 1 \mathrm{a}) \\
C(t, R) & =C_{o} & (\mathrm{~S} 1 \mathrm{~b}) \\
\left.\frac{\partial C}{\partial r}\right|_{r=R_{b}}=0 & (\mathrm{~S} 1 \mathrm{c})
\end{array}
$$

Equation S1 was non-dimensionalized using the defined variables shown in equations S2a-c. The non-dimensional version of the equation S1 is shown in equation S3, with the corresponding non-dimensional initial and boundary conditions in equations S3a-c.

$$
\bar{C}=\frac{C}{C_{o}}
$$




$$
\begin{gathered}
\bar{r}=\frac{r-R}{R_{b}-R} \quad(\mathrm{~S} 2 \mathrm{~b}) \\
\tau=\frac{t D}{\left(R_{b}-R\right)^{2}} \quad(\mathrm{~S} 2 \mathrm{c}) \\
\frac{\partial \bar{C}}{\partial \tau}=\frac{\partial^{2} \bar{C}}{\partial \bar{r}^{2}}, \tau \in[0, \infty], \bar{r} \in[0,1] \\
\bar{C}(0, \bar{r})=C_{1} / C_{o} \\
\bar{C}(\tau, 0)=1 \\
\left.\frac{\partial \bar{C}}{\partial \bar{r}}\right|_{\bar{r}=1}=0
\end{gathered}
$$

To make the boundary conditions homogenous, a non-dimensional variable $U$ was defined, as shown in equation S4. By substituting equation S4 into equation S3, equation S5 was obtained. The corresponding initial and boundary conditions are shown in equations S5a-c.

$$
\begin{gathered}
U(\tau, \bar{r})=\bar{C}(\tau, \bar{r})-1 \\
\frac{\partial U}{\partial \tau}=\frac{\partial^{2} U}{\partial \overline{r^{2}}} \\
U(0, \bar{r})=\frac{C_{1}}{C_{o}}-1 \\
U(\tau, 0)=0 \\
\left.\frac{\partial U}{\partial \bar{r}}\right|_{\bar{r}=1}=0
\end{gathered}
$$

Using separation of variables, "U" can be defined as the product of two functions; a $\tau$-dependent function $T(\tau)$ and an $\bar{r}$-dependent function $R \overline{(r}$ ) (equation 6). The corresponding boundary conditions are shown in equations S6a-b. Equation S6c was obtained by substituting equation S6 into equation S5.

$$
\begin{gathered}
U=T(\tau) R(\bar{r}) \\
T(\tau) R(0)=0 \\
T(\tau) R^{\prime}(1)=0
\end{gathered}
$$




$$
T^{\prime} R=R^{\prime \prime} T \quad(\mathrm{~S} 6 \mathrm{c})
$$

$\mathrm{T}$ and $\mathrm{R}$ were separated into separate functions which must both equal a constant $\left(-\lambda^{2}\right)$ as shown in equation S7. The corresponding ordinary differential equations for $\mathrm{T}$ and $\mathrm{R}$ are represented in equations S8 and S9, respectively. Due to homogeneity of the boundary conditions, boundary conditions for equation S9 were obtained and are shown in equations S9a and S9b.

$$
\begin{gathered}
\frac{T^{\prime}}{T}=\frac{R^{\prime \prime}}{R}=-\lambda^{2} \\
T^{\prime}+\lambda^{2} T=0 \\
R^{\prime \prime}+\lambda^{2} R=0 \\
R(0)=0 \\
R^{\prime}(1)=0
\end{gathered}
$$

The general solution to equation S9 is shown below in equation S10. The boundary conditions were used to solve for the constants of integration. Using the boundary condition from equation $\mathrm{S} 9 \mathrm{a}$, it can be seen that $\mathrm{c}_{2}$ is equal to 0 . When applying the boundary condition from equation $\mathrm{S} 9 \mathrm{~b}$, equation $\mathrm{S} 10 \mathrm{a}$ was obtained. To avoid a trivial solution, $\mathrm{c}_{1}$ cannot equal 0 , therefore $\lambda$ corresponds to the eigenvalues of the Sturm-Louisville problem $\left(\lambda_{n}\right)$, which is defined in equation S10b. Equation S10c is the solution to the R-problem. The general solution to the Tproblem (equation S8) is shown in equation S10d.

$$
\begin{gathered}
R=c_{1} \sin (\lambda \bar{r})+c_{2} \cos (\lambda \bar{r}) \\
0=c_{1} \cos (\lambda \bar{r}) \\
\lambda=\lambda_{n}=\pi\left(\frac{2 n+1}{2}\right), n=0,1,2,3 \ldots . \\
R=c_{1} \sin \left(\lambda_{n} \bar{r}\right) \\
T=c_{3} e^{-\lambda_{n}^{2 \tau}}
\end{gathered}
$$

Equations S10c and S10d were substituted into equation S6. Superposition of the infinite 
eigenvalues results in an infinite summation solution as depicted in equation $\mathrm{S} 11 . \mathrm{C}_{\mathrm{n}}$ represents the combined constants of integration $\left(\mathrm{c}_{1} *^{*} \mathrm{c}_{3}\right)$ and is dependent upon " $\mathrm{n}$ ". By substituting the initial condition (Equation S5a), equation S11a was obtained. From orthogonality of sine functions, it is known that integration over the boundary range (0-1) of two sine functions with identical eigenvalues is equal to $1 / 2$ (equation S11b). When the eigenvalues differ, the integrated product is equal to 0 . Therefore, both sides of equation $\mathrm{S} 11$ were multiplied by $\sin \left(\lambda_{\mathrm{m}}\right)$ and integrated from 0-1, resulting in equation S11c. The solution shown in equation S11d was obtained when $\mathrm{m}=\mathrm{n}$.

$$
\begin{gathered}
U=\sum_{n=0}^{\infty} C_{n} e^{-\lambda_{n}{ }^{2} \tau} \sin \left(\lambda_{n} \bar{r}\right) \\
\frac{C_{1}}{C_{o}}-1=\sum_{n=0}^{\infty} C_{n} \sin \left(\lambda_{n} \bar{r}\right) \quad \text { (S11a) } \\
\int_{0}^{1} \sin \left(\lambda_{m} \bar{r}\right) \sin \left(\lambda_{n} \bar{r}\right) d \bar{r}=\left\{\begin{array}{cc}
1 / 2 & \text { when } m=n \\
0 \quad \text { when } m \neq n
\end{array}\right. \\
\left(\frac{C_{1}}{C_{o}}-1\right) \int_{0}^{1} \sin \left(\lambda_{m} \bar{r}\right) d \bar{r}=\sum_{n=0}^{\infty} C_{n} \int_{0}^{1} \sin \left(\lambda_{m} \bar{r}\right) \sin \left(\lambda_{n} \bar{r}\right) d \bar{r} \\
C_{n}=\frac{2\left(\frac{C_{1}}{C_{o}}-1\right)}{\lambda_{n}} \quad \text { (S11d) }
\end{gathered}
$$

Equation S11d was substituted into equation S11, resulting in equation S12. The variable "U" was then substituted with " $\bar{C}-1$ ", and the equation was dimensionalized, resulting in the concentration profile shown in equation S13.

$$
\begin{gathered}
U=2\left(\frac{C_{1}}{C_{o}}-1\right) \sum_{n=0}^{\infty} \frac{1}{\lambda_{n}} e^{-\lambda_{n}^{2} \tau} \sin \left(\lambda_{n} \bar{r}\right) \\
C(t, r)=C_{o}+2 C_{o}\left(\frac{C_{1}}{C_{o}}-1\right) \sum_{n=0}^{\infty}\left[e^{-\frac{\lambda_{n}^{2} D t}{\left(R_{b}-R\right)^{2}}} \sin \left(\frac{\lambda_{n}(r-R)}{R_{b}-R}\right)\left(\frac{1}{\lambda_{n}}\right)\right]
\end{gathered}
$$

The initial domain radius $\left(R(0)=R_{0}\right)$ was determined by calculating the average area per domain 
(i.e. dividing the total area of domains by the number of domains), dividing by $\pi$ and taking the square root. $\mathrm{R}_{\mathrm{b}}$ was determined through a similar method (i.e. dividing the total area of the multibilayer by the number of domains, but multiplying by 0.9069 to account for circle packing ${ }^{1}$, dividing by $\pi$ and taking the square root).

Partition Coefficient Derivation. A mass balance was performed on a single domain within a dissolution region with radius $\mathrm{R}_{b}$ as shown below in Fig. S1. When the $\mathrm{L}_{o}$ domain begins to dissolve, a mixing zone approximated by an annulus with thickness $\mathrm{R}_{\text {mix }}-\mathrm{R}$ is formed. As the dissolution proceeds, the mixing annulus increases in thickness. When complete dissolution occurs, $R_{\text {mix }}$ would be equal to $R_{b}$ (the radius of the boundary). When no dissolution occurs, $R_{\text {mix }}$ is equal to zero (i.e. $\mathrm{R}_{\text {mix }}=\mathrm{R}=\mathrm{R}_{\mathrm{o}}$ ). Therefore, the area of the mixing annulus is directly proportional to the area decrease of the $\mathrm{L}_{\mathrm{o}}$ domain, as shown in equation S14. In other words, the fractional decrease in domain area $\left(1-\mathrm{R}^{2} / \mathrm{R}_{0}{ }^{2}\right)$ multiplied by the total area of the annulus between $R_{b}$ and $R$ is equal to the area of the mixing annulus.
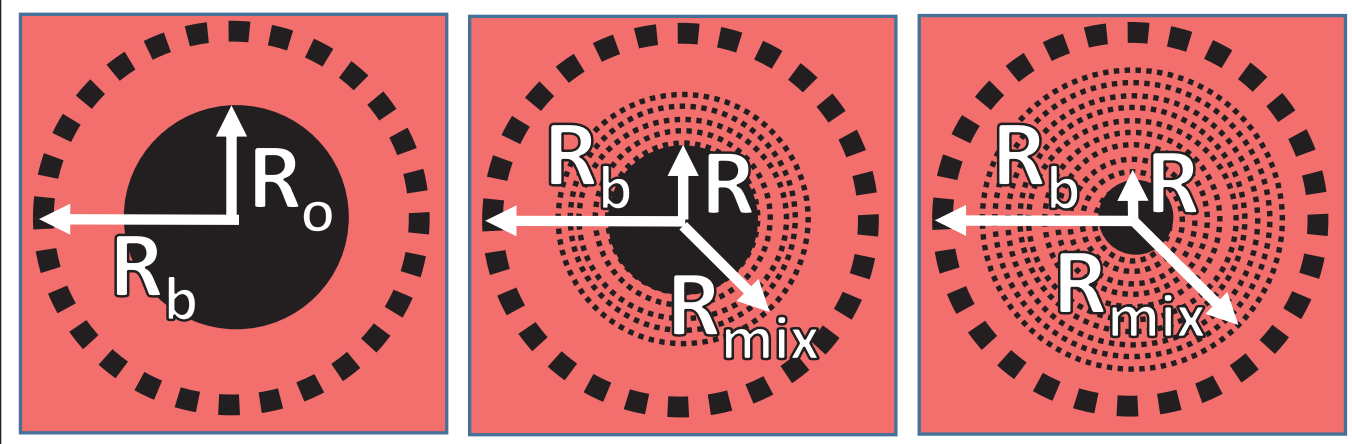

Time

Figure S1: Growing mixing annulus between $\mathrm{L}_{\mathrm{o}}$ domain and $\mathrm{L}_{\mathrm{d}}$ region. The annulus is composed of both $\mathrm{L}_{\mathrm{o}}$ and $\mathrm{L}_{\mathrm{d}}$ lipids. As the mixing annulus increases, the $\mathrm{L}_{\mathrm{o}}$ domain and $\mathrm{L}_{\mathrm{d}}$ region both decrease in size. 


$$
\operatorname{Area}_{\text {mix }}=\pi\left(R_{\text {mix }}^{2}-R^{2}\right)=\pi\left(1-\frac{R^{2}}{R_{o}^{2}}\right)\left(R_{b}^{2}-R^{2}\right)
$$

Assuming the number of lipids in a phase is proportional to its area, the partition coefficient $(\mathrm{K})$ can be defined as the ratio of unmixed area to mixed area as shown in equation S15. Unmixed area equals the combined area of remaining $\mathrm{L}_{\mathrm{o}}$ domain and $\mathrm{L}_{\mathrm{d}}$ phase, which is shown in equation S16 (i.e. the total area of the region minus the area of the mixing annulus). Therefore, equation S17 was obtained by substituting equations S14 and S16 into equation S15.

$$
\begin{gathered}
K=\frac{N_{\text {unmix }}}{N_{\text {mix }}}=\frac{\text { Area }_{\text {unmix }}}{\text { Area }_{\text {mix }}} \\
\text { Area }_{\text {unmix }}=\pi R_{b}^{2}-\pi\left(1-\frac{R^{2}}{R_{o}^{2}}\right)\left(R_{b}^{2}-R^{2}\right) \\
K=\frac{R_{b}^{2}-\left(1-\frac{R^{2}}{R_{O}^{2}}\right)\left(R_{b}^{2}-R^{2}\right)}{\left(1-\frac{R^{2}}{R_{O}^{2}}\right)\left(R_{b}^{2}-R^{2}\right)}
\end{gathered}
$$

Since $\left(R_{0} / R_{b}\right)^{2}$ is equal to the initial area fraction $\left(A F_{0}\right)$ and $\left(R / R_{b}\right)^{2}$ is equal to the area fraction at any given time (AF), the numerator and denominator in equation $\mathrm{S} 17$ was multiplied by $\left(1 / \mathrm{R}_{\mathrm{o}}{ }^{2}\right)$ to obtain equation S18. Using this form, the partition coefficient can be calculated via area fraction measurements.

$$
K=\frac{\frac{1}{A F_{O}}-\left(1-\frac{A F}{A F_{O}}\right)\left(\frac{1}{A F_{O}}-\frac{A F}{A F_{O}}\right)}{\left(1-\frac{A F}{A F_{O}}\right)\left(\frac{1}{A F_{O}}-\frac{A F}{A F_{O}}\right)}
$$

Activation Energy Determination. The activation energy associated with lipids transferring from a $\mathrm{L}_{\mathrm{o}}$ domain to the surrounding $\mathrm{L}_{\mathrm{d}}$ region was calculated using the Arrhenius equation, shown below in equation S19. First order reaction rate constants $(\mathrm{k})$ were determined for $12 \%$ DPIDA domains bound with GFP, NLP1, and NLP3. Since the relative reaction rates between samples are being compared, it is not necessary to determine an exact reaction order. This point is augmented by the assumption that the reaction mechanism should not vary between samples, 
as the only thing changing is the size of the binding particle (i.e. magnitude of steric pressure). Rate constants were obtained by regressing the relative area fraction $\left(\mathrm{AF} / \mathrm{AF}_{0}\right)$ vs time data in Fig. 3 of the manuscript to a first-order exponential decay model, shown below in equation S19a. The values of $\mathrm{k}$ determined for GFP, NLP1, and NLP3 were 0.012, 0.014, and $0.083 \mathrm{sec}^{-1}$, respectively.

$$
\begin{gathered}
k=A e^{-\frac{E_{A}}{k_{B} T}} \\
\left(\frac{A F}{A F_{o}}\right)=e^{-k t}
\end{gathered}
$$

The change in activation energy $\left(\Delta \mathrm{E}_{\mathrm{A}}=\mathrm{E}_{\mathrm{A} 2}-\mathrm{E}_{\mathrm{A} 1}\right)$ is shown in equation S20. When comparing GFP to NLP1, NLP1 resulted in an activation energy reduction $\left(\Delta \mathrm{E}_{\mathrm{A}}\right)$ of $4.4 \times 10^{-22}$ Joules/lipid $(0.1 \mathrm{kT})$. When comparing NLP1 to NLP3, NLP3 resulted in an activation energy reduction of $7.3 \times 10^{-21}$ Joules/lipid (1.8kT).

$$
\Delta E_{A}=k_{B} T * \ln \left(\frac{k_{1}}{k_{2}}\right)
$$

By looking at $\Delta \mathrm{E}_{\mathrm{A}}$, the order of magnitude of initial activation energy (i.e. no particles bound) could be estimated. When bound with GFP and NLP1, $\mathrm{L}_{0}$ domain dissolution appeared to be reaction limited. When bound with NLP3, the process appeared to be diffusion limited. Therefore with NLP3 binding, the relative reaction rate must have been in the proximity of 1 , while in the GFP/NLP1 cases, it was significantly lower. When looking at Fig. S2, it can be seen that the relative reaction rate changes by an order of magnitude when the activation energy varies from $0-2 \mathrm{kT}$. If $\mathrm{E}_{\mathrm{A}}$ is in the range of $1-2 \mathrm{kT}$, a $0.1 \mathrm{kT}$ reduction (i.e. difference in GFP and NLP1 binding) does not significantly alter the relative reaction rate. However, a $1.8 \mathrm{kT}$ reduction (i.e. difference in NLP1 and NLP3 binding) does. Since this theoretical behavior is consistent with the observed behavior, it is strongly suggested that the actual activation energy is on the order of 


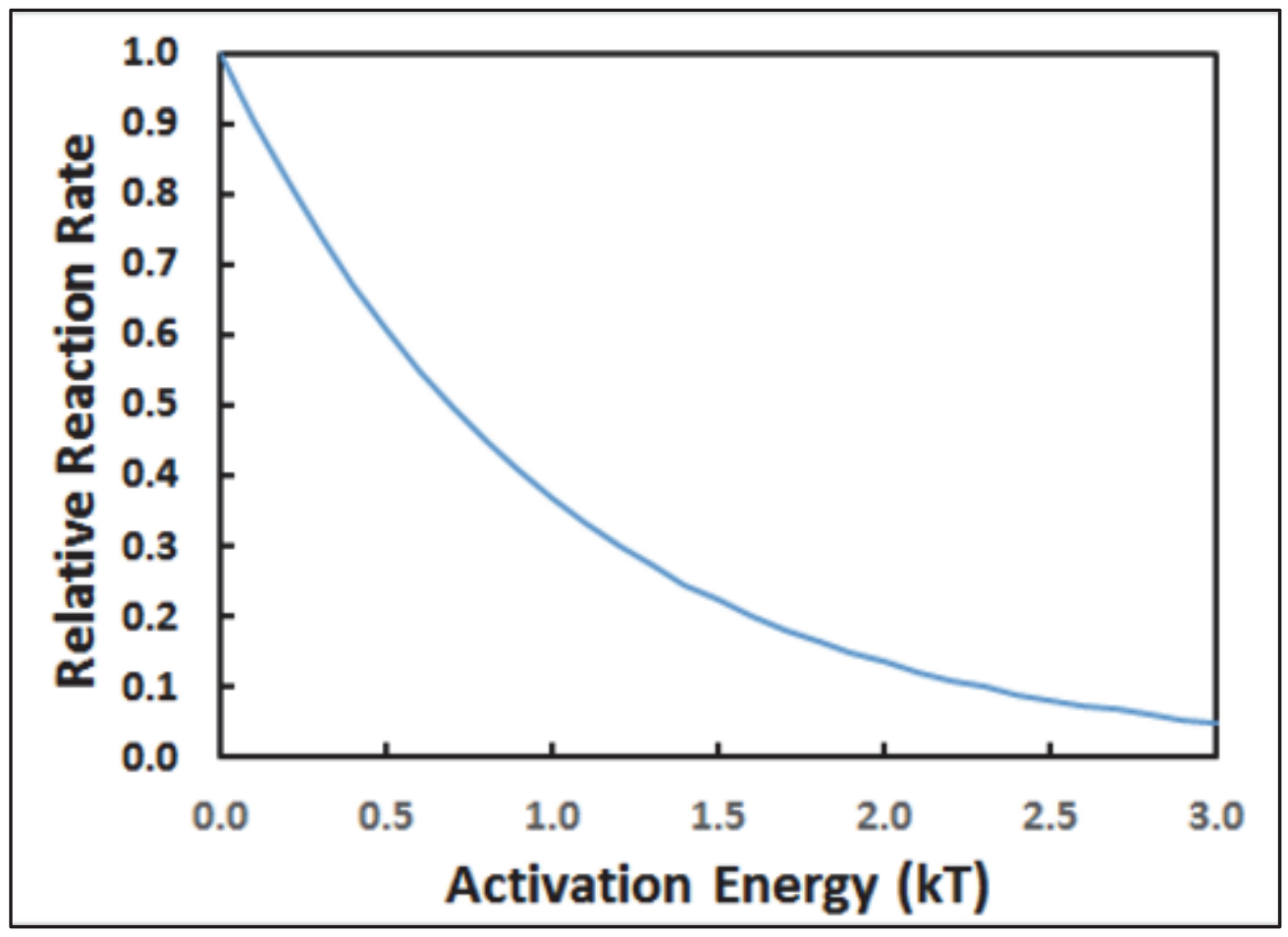

Figure S2: Relative reaction rate as a function activation energy normalized by kT. This relationship was determined using the Arrhenius Equation.

Steric Pressure Contribution to Free Energy. The Carnahan-Starling equation of state is depicted below in equation S21. ${ }^{2}$ The surface coverage, $\eta$, is the fractional coverage of proteins/particles on a bilayer surface (equation S22). ${ }^{3-4}$ It is the number of particles $\left(\mathrm{N}_{\mathrm{P}}\right)$, multiplied by the area of each particle $\left(A_{P}\right)$, divided by the total area of bilayer in which IDA lipids are present $\left(A_{c}\right)$. The volume $\left(V_{c}\right)$ is simply the total area of bilayer multiplied by the bilayer height (i.e. $\mathrm{A}_{\mathrm{c}}$ *height).

$$
\begin{gathered}
P=\frac{N_{P} k_{B} T}{V_{c}}\left(\frac{1+\eta+\eta^{2}-\eta^{3}}{[1-\eta]^{3}}\right) \\
\eta=\frac{N_{P} A_{P}}{A_{c}}
\end{gathered}
$$


The free energy contribution from steric pressure $\left(\Delta G_{p}\right)$ is shown below in equation $S 23$, where it is demonstrated that a dissolving $\mathrm{L}_{\mathrm{o}}$ domain is analogous to the work done by an expanding gas. ${ }^{3}$ By solving equation S22 for $A_{c}$ and substituting it (along with equation S21) into equation S23, equation S24 was obtained. The factor $1 / \mathrm{N}_{\mathrm{L}}$ on the outside of the integrand allows for normalization of the free energy to a per lipid basis.

$$
\begin{gathered}
\Delta \mathrm{G}_{\mathrm{p}}=-\int_{V_{i}}^{V_{f}} P d V_{c} \\
\Delta G_{p}=\frac{1}{N_{L}} \int_{\eta_{i}}^{\eta_{f}} N_{P} k_{B} T\left(\frac{1+\eta+\eta^{2}-\eta^{3}}{\eta[1-\eta]^{3}}\right) d \eta
\end{gathered}
$$

Values of $\eta_{\mathrm{f}}$ and $\eta_{\mathrm{i}}$ were calculated using equation S25. It was assumed that histidine-tags on average bind to 2-3 (i.e. 2.5) IDA lipids at a time. ${ }^{4}$ Ubiquitin, GFP, NLP1, and NLP3 were all assumed to have one binding histidine tag per protein/particle. The final $\eta\left(\eta_{f}\right)$ was calculated using a mol\% DPIDA corresponding to its overall mole fraction, whereas the initial $\eta\left(\eta_{\mathrm{i}}\right)$ was calculated using a mol\% DPIDA corresponding to its mole fraction relative to (DPPC + DPIDA). For NLP1, a limiting packing fraction $\left(\eta_{\max }\right)$ of 0.92 was used when calculated values were between $0.92-1.0 .^{3}$

$$
\eta=\frac{(\text { mol\%DPIDA }) *\left(\frac{1 \text { Particle }}{2.5 \text { DPIDA }}\right) *\left(\frac{\text { Area Particle }}{1 \text { Particle }}\right)}{\left[(1-\text { mol\%DPIDA }) *\left(\frac{\text { Area Lipid }}{1 \text { Lipid }}\right)\right]+\left[(\text { mol\%DPIDA }) *\left(\frac{1 \text { Particle }}{2.5 \text { DPIDA }}\right) *\left(\frac{\text { Area Particle }}{1 \text { Particle }}\right)\right]}
$$

Saffman-Delbrück Model for Determining Decrease in Diffusivity. The Saffman-Delbrück is an appropriate model for calculating the diffusion coefficient of species within a lipid bilayer. ${ }^{5}$ The diffusion coefficient is shown in equation S26, where $\mu_{\mathrm{m}}$ and $\mu_{\mathrm{f}}$ correspond to the viscosities of the membrane and surrounding fluid, respectively. The bilayer height is denoted by "h", while the cylindrical radius of the diffusing species is denoted by "a". 


$$
D=\frac{k_{B} T}{4 \pi \mu_{m} h}\left[\ln \left(\frac{h \mu_{m}}{a \mu_{f}}\right)-0.577\right]
$$

Values for $\mu_{\mathrm{m}}$ and $\mu_{\mathrm{f}}$ were chosen to be $5 \times 10^{-1}$ and $1 \times 10^{-3} \mathrm{~Pa}^{*} \mathrm{~s}$, respectively. The bilayer height " $\mathrm{h}$ " was chosen to be $5 \mathrm{~nm}$, while the cylindrical radius "a" is defined in equation $\mathrm{S} 27 . \mathrm{N}_{\mathrm{L}}$ and $A_{L}$ correspond to the number of lipids and area of a single lipid, respectively. $A_{L}$ was chosen to be $0.6 \mathrm{~nm}^{2}$.

$$
a=\sqrt{\frac{N_{L} A_{L}}{\pi}}
$$

Using these specified values, $D$ is equal to $1 \mu \mathrm{m}^{2} / \mathrm{s}$ when $\mathrm{N}_{\mathrm{L}}$ is chosen to be 1 . When $\mathrm{N}_{\mathrm{L}}$ is equal to $10^{3}$ lipids, D is equal to $0.6 \mu \mathrm{m}^{2} / \mathrm{s}$. This value decreases to $0.4 \mu \mathrm{m}^{2} / \mathrm{s}$ when $10^{4}$ lipids are used.

Processing of Images for Area Fraction Determination. Area fractions were determined using the program ImageJ. Microscope Images (Fig. S3A) were converted to binary black and white images using an appropriate color threshold for each image (Fig. S3B). Afterward, domains were traced (Fig. S3C) using an appropriate size cut-off. The total area inside of these traces divided by the total area of the image is equal to the area fraction of domains. Sample processed images are depicted for NLP1 binding to 12\% DPIDA multibilayers in Fig. S3. 


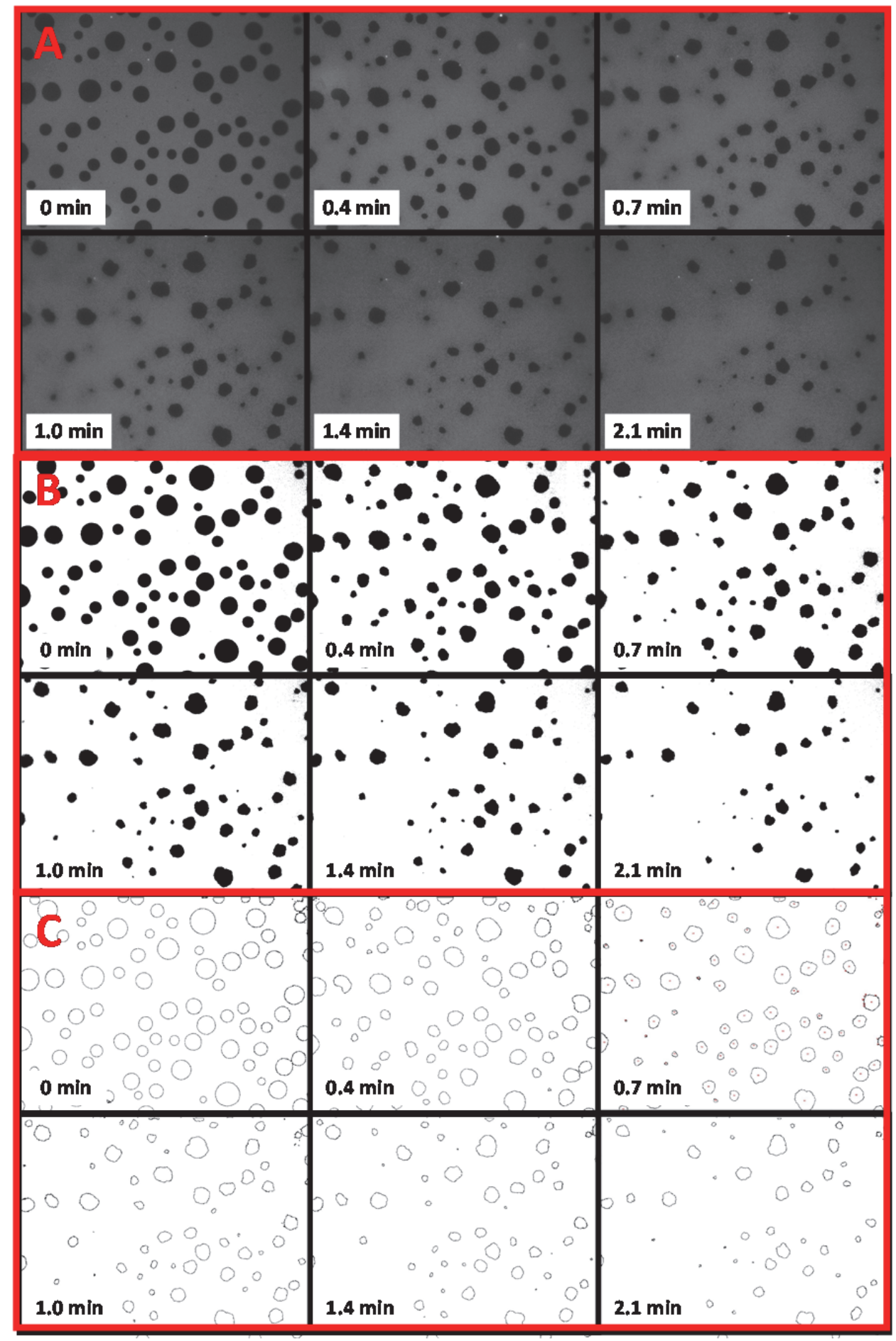

Figure S3: Microscope images of NLP1 binding to 12\% DPIDA MBLs (A), converted to binary black and white images (B), and traced (C). 


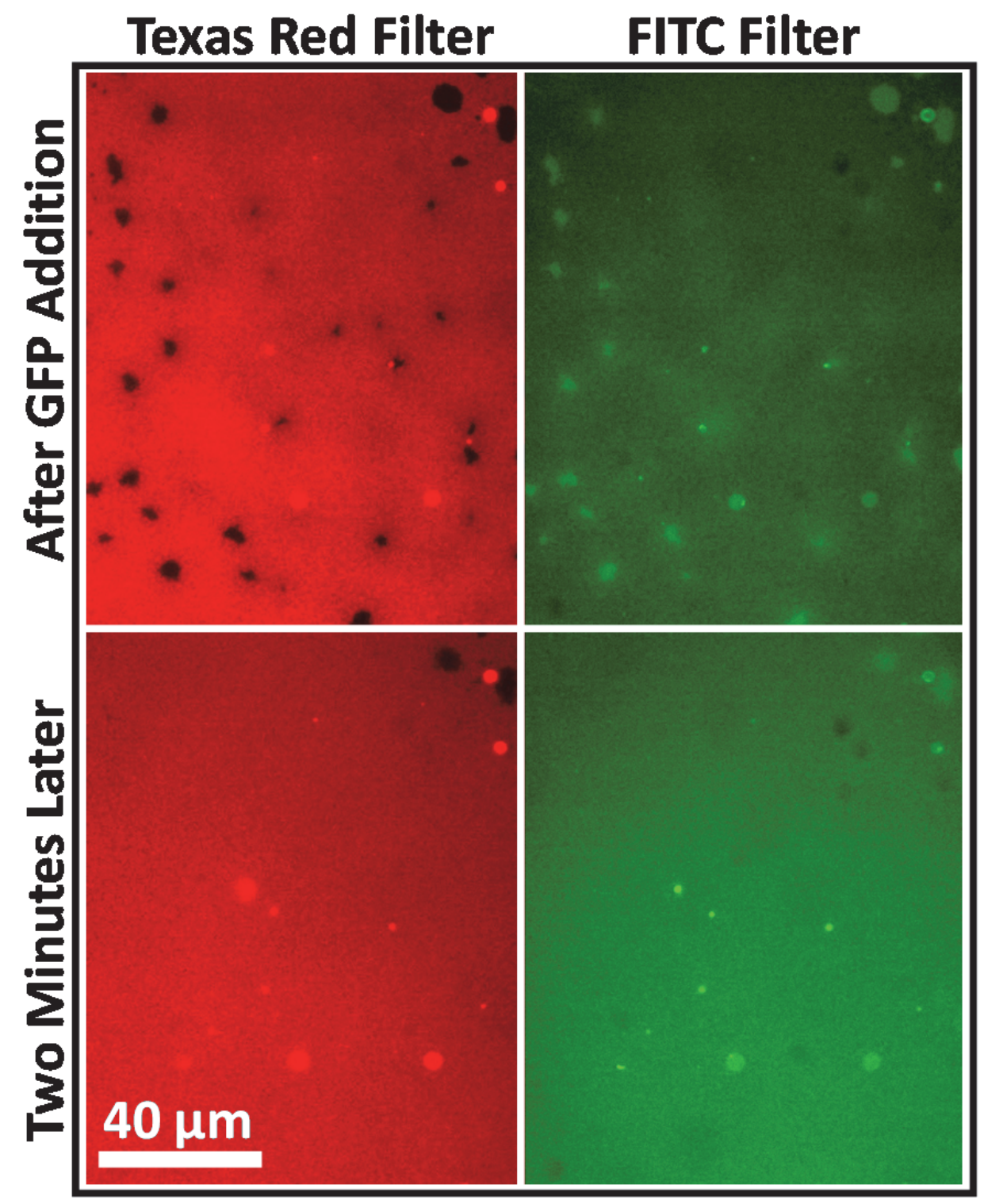

Figure S4: Dissolution of $\mathrm{L}_{\mathrm{o}}$ domains in MBLS induced by GFP. The process was visualized in both Texas Red and FITC filter channels. The MBL composition was 49.9/20/12/18/0.1 mol\% DOPC/DPPC/DPIDA/Cholesterol/Texas Red-DHPE. 




Figure S5: Binding of Ubiquitin to $\mathrm{L}_{\mathrm{o}}$ domains in multibilayers. There is an initial formation of additional, smaller domains (A-B), followed by incomplete dissolution and coalescence of domains (C-D). The multibilayer composition was 49.9/20/12/18/0.1 mol\% DOPC/DPPC/DPIDA/Cholesterol/Texas Red-DHPE. 


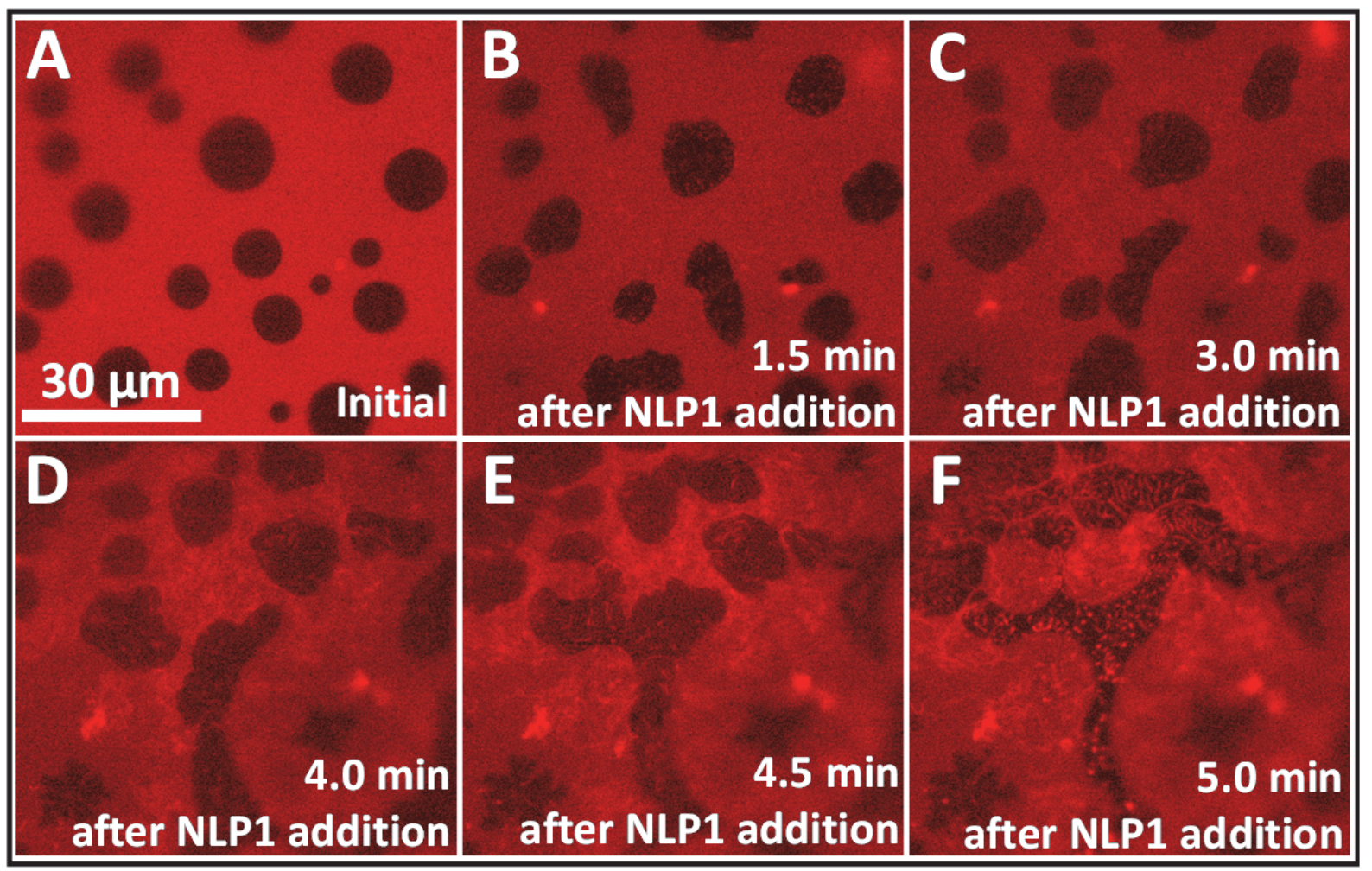

Figure S6: Binding of NLP1 to the $\mathrm{L}_{\mathrm{d}}$ region of MBLs containing 29.9/32/20/18/0.1 mol\%

DOPC/DPPC/DOIDA/Cholesterol/Texas Red-DHPE. Mixing was allowed to proceed for a

longer duration (A-F) than illustrated in Fig. 6 of the manuscript. $\mathrm{L}_{\mathrm{d}}$ domains can be seen within elongated $\mathrm{L}_{\mathrm{o}}$ domains $(\mathrm{F})$.

Table S1: Parameters for $\mathrm{L}_{\mathrm{o}}$ Domain dissolution used in Equation 4. D and $\mathrm{C}_{1} / \mathrm{C}_{\mathrm{o}}$ were regressed, while $R_{o}$ and $R_{b}$ were determined from image analysis.

\begin{tabular}{|c|c|c|c|c|c|c|c|}
\hline & & & & Mir & & Dem & ring \\
\hline & & $\begin{array}{c}\text { Initial } \\
\text { Domain } \\
\text { Radius } \\
\left(\mathbf{R}_{0}\right)(\mu \mathrm{m})\end{array}$ & $\begin{array}{c}\text { Initial } \\
\text { Boundary } \\
\text { Radius } \\
\left(\mathbf{R}_{\mathrm{b}}\right)(\mu \mathrm{m})\end{array}$ & $\begin{array}{l}\text { Diffusion } \\
\text { Coefficient } \\
\text { (D) }\left(\mu \mathrm{m}^{2} / \mathrm{s}\right)\end{array}$ & $\mathrm{C}_{1} / \mathrm{C}_{\mathrm{o}}$ & $\begin{array}{l}\text { Diffusion } \\
\text { Coefficient } \\
\text { (D) }\left(\mu \mathrm{m}^{2} / \mathrm{s}\right)\end{array}$ & $\mathrm{C}_{1} / \mathrm{C}_{\mathrm{o}}$ \\
\hline & Ubiq & 3.3 & 6.1 & - & - & - & - \\
\hline$\theta^{0}$ & GFP & 2.4 & 4.9 & $0.02+0.01$ & $0.35+0.10$ & $0.15+0.06$ & $0.24+0.09$ \\
\hline & NLP1 & 3.8 & 7.9 & $0.06+0.01$ & $0.31+0.04$ & $0.55+0.20$ & $0.24+0.11$ \\
\hline & NLP3 & 3.6 & 7.9 & $0.79+0.08$ & $0.52+0.01$ & $0.65+0.22$ & $0.31+0.05$ \\
\hline & Ubiq & 1.9 & 4.1 & - & - & - & - \\
\hline$\therefore \overparen{\theta}$ & GFP & 2.6 & 5.2 & - & - & - & - \\
\hline & NLP1 & 3.9 & 8.4 & $0.06+0.01$ & $0.50+0.01$ & $0.62+0.13$ & $0.44+0.05$ \\
\hline & NLP3 & 3.3 & 6.0 & $0.18+0.05$ & $0.42+0.12$ & $0.55+0.11$ & $0.28+0.04$ \\
\hline
\end{tabular}




\section{REFERENCES}

(1) Goldberg, M. The Packing of Equal Circles in a Square. Math. Mag. 1970, 43, 24-30.

(2) Carnahan, N. F.; Starling, K. E. Equation of State for Nonattracting Rigid Spheres. J. Chem. Phys. 1969, 51, 635-636.

(3) Zeno, W. F.; Rystov, A.; Sasaki, D. Y.; Risbud, S. H.; Longo, M. L. Crowding-Induced Mixing Behavior of Lipid Bilayers: Examination of Mixing Energy, Phase, Packing Geometry, and Reversibility. Langmuir 2016, 32, 4688-4697.

(4) Scheve, C. S.; Gonzales, P. A.; Momin, N.; Stachowiak, J. C. Steric Pressure between Membrane-Bound Proteins Opposes Lipid Phase Separation. J. Am. Chem. Soc. 2013, 135, 1185-1188.

(5) Saffman, P.; Delbrück, M. Brownian Motion in Biological Membranes. Proceedings of the National Academy of Sciences 1975, 72, 3111-3113. 